\title{
Similarity transformations for the two-dimensional, unsteady, stream-function equation
}

\author{
BY BRIAN J. CANT WELL
}

\author{
Graduate Aeronautical Laboratories, \\ California Institute of Technology, Pasadena
}

(Received 2 March 1977 and in revised form 12 July 1977)

The methods described by Bluman \& Cole (1974) are used to derive the infinitesimals of the general invariance group of the unsteady, two-dimensional, stream-function equation for the case where the kinematic viscosity $\nu$ is equal to a constant and the case where $\nu=0$. The infinitesimals in each case involve ten independent parameters, seven of which appear explicitly and three of which are contained implicitly in three arbitrary functions of time. The various finite groups and similarity transformations which may be derived from the infinitesimals are discussed through examples. Two of the arbitrary functions of time are non-trivial and represent invariance of the streamfunction equation under a transformation to a co-ordinate system which moves in a non-uniform irrotational fashion. A general similarity form is derived for which the equations $d x / d t=u(x, y, t)$ and $d y / d t=v(x, y, t)$ for the particle paths may be reduced to an autonomous system. This form is general enough to suggest the hypothesis that, under certain restrictions, the entrainment processes of unsteady flows dominated by two-dimensional large-scale motions may be displayed diagrammatically on a phase-plane plot of particle trajectories.

\section{Introduction}

It has been known for some time that the process of Reynolds averaging hides from view many of the salient features of turbulent flow. There is now strong evidence which suggests that a wide variety of turbulent shear flows, once thought to consist of undifferentiated masses of randomly moving, vorticity-bearing fluid elements, are actually dominated by large-scale, often two-dimensional, unsteady motions which are not random. The most notable example is the experiment by Brown \& Roshko (1974), who discovered large eddies in a fully developed mixing layer. The effect of this work has been to motivate a renewed interest in the general problem of the unsteady flow of an incompressible fluid in two dimensions.

There are several corollaries to the coherent-eddy picture of turbulent shear flow. To begin with, the problem becomes much more complicated since any description of the flow must include the fundamental unsteadiness. Furthermore, since instantaneous velocity fluctuations in the $x$ and $y$ directions may be of the same order, the traditional boundary-layer approach must be replaced by an examination of the complete equations of motion. On the other hand, since the eddies are convected by the main flow, one might hope to simplify things a bit by moving in a co-ordinate system whose origin was somehow fixed to an eddy. Lastly the message which comes through in the 
photographs of the mixing layer by Brown \& Roshko (1974), the photographs of the wake by Zdravkovich (1969), photographs of the early region of jets and many other pictures of unsteady fluid flows is the message of symmetry. The existence of an array of vortex structures, given certain boundary conditions and the requirement of continuity, imposes certain compatibility conditions on the flow. Entrainment of free-stream fluid occurs as a natural consequence of the induced motions of the large eddies and the compatibility conditions are manifested in terms of points of fluid accumulation. For example, when the velocity field in the near wake of a circular cylinder is viewed from a frame of reference moving with a vortex (Cantwell 1975) the result is a slowly varying pattern of centres and saddles.

This paper is concerned, in one way or another, with each of the above corollaries. To begin with, there is a growing number of experimenters, sometimes called eddy chasers, $\dagger$ who are trying to map large eddy motions in space as well as time; see, for example, Browand (1975, mixing-layer measurements), Fiedler (1975, mixing-layer measurements), Cantwell (1975, cylinder near-wake measurements), Cantwell, Coles \& Dimotakis (1977, turbulent-spot measurements), Coles \& Barker (1975, turbulentspot measurements), Wygnanski, Sokolov \& Friedman (1976, turbulent-spot measurements) and Turner (1964, turbulent spherical-vortex model). The eddy chasers face the practical problem of collapsing onto a two-dimensional plot measurements or computations made in a three-dimensional space; there is a need for finding similarity transformations which reduce the number of independent variables in the problem. Furthermore, the velocity field, which is the quantity one is usually concerned with, depends on the motion of a moving frame of reference and therefore may lead to conflicting or erroneous conclusions when used to argue the disposition of the large eddies; there is a need for understanding the relationship between the frame of reference of a moving observer and the unsteady flow being observed. Finally, the entrainment process, which is an essential part of literally all unconfined turbulent shear flows, can properly be described only in terms of particle paths, the computation of which may present formidable analytical difficulties even if the unsteady velocity field is known exactly (Hama 1962). We may hypothesize that approximating the flow field by a similarity form might prove helpful in this regard even if the similarity were not exact, but could be applied only over a limited space, of the order of one or two eddy lengths, and/or for a limited period of time, comparable to the lifetime of an eddy. To see how this might work, imagine an observer moving in a co-ordinate system with origin attached to the centre of an eddy defined, say, by a peak in the vorticity field or a minimum in the pressure field. In such a system the flow, locally, would be quasisteady; the eddy would be growing in size and, for times small compared with its lifetime, might remain relatively undistorted by neighbouring eddies. If one could fit a similarity form to the stream function over this limited region of the flow, then the problem of computing particle paths might be considerably simplified. The possibilities of this approach to the entrainment problem will be addressed in the latter part of this paper.

First, the problem of proper frames of reference and the symmetry properties of two-dimensional unsteady flows is approached through the theory of Lie groups. The

+ Invariably the eddy chaser is a cartoonist whose sketches of the whorls and convolutions of vortex flows are at once informative because they show how the flow must go and frustrating because they defy definition. This paper describes a possible escape from this dilemma. 
methods described by Bluman \& Cole (1974) are used to derive the infinitesimals of the general invariance group of the stream-function equation for the case where the kinematic viscosity $\nu$ is equal to a constant and the case where $\nu=0$ (Euler's equation). The infinitesimals in each case involve ten independent parameters, seven of which appear explicitly and three of which are contained implicitly in three arbitrary oncedifferentiable functions of time. The various finite groups implied by the infinitesimals are discussed. Two of the arbitrary functions of time contained in the infinitesimals are non-trivial and represent invariance of the stream-function equation under a transformation to a co-ordinate system which moves in a non-uniform irrotational fashion. The infinitesimals are used to derive a few of the possible similarity transformations which the equation will admit, and for one rather general case it is shown that the equations for the unsteady particle paths may be reduced to an autonomous system.

The reader who is unfamiliar with group theory may wish to refer to the introductory works of Birkhoff (1950), Hansen (1964) and especially Cohen (1911). A thorough treatment of group methods using infinitesimal transformations may be found in Ovsjannikov (1962) and a comparison of methods using finite and infinitesimal transformations may be found in Woodard \& Ames (1971). Ames (1972) contains a survey of group methods, along with a valuable bibliography on the subject. Transformation techniques have been applied to related problems by Strumpf (1964) and Rosen \& Ullrich (1973).

\section{The problem}

The motion of an incompressible constant-property fluid in two dimensions is described by the stream-function equation

$$
\nabla^{2} \psi_{t}+\psi_{y} \nabla^{2} \psi_{x}-\psi_{x} \nabla^{2} \psi_{y}=\nu \nabla^{\mathbf{4}} \psi \quad\left(\nabla^{2} \equiv \partial^{2} / \partial x^{2}+\partial^{2} / \partial y^{2}\right)
$$

where the stream function $\psi$ is defined by $u=\partial \psi / \partial y$ and $v=-\partial \psi / \partial x$ in order to satisfy the continuity equation identically. We wish to compute the various transformation groups which leave the functional form of (1) invariant. The infinitesimals derived from these groups may be used to find similarity transformations of the form

$$
\psi=g(x, y, t, \theta(x, y, t), \phi(x, y, t), F(\theta, \phi)),
$$

which, when substituted into (1), lead to a new partial differential equation for $F$ in terms of $\theta$ and $\phi$, and thus to a reduction in the number of independent variables from three to two.

The application of such transformations to specific cases requires that the boundary curves and the functions of $\psi$ specified on them satisfy the same conditions of invariance. However, the effort in this paper will be restricted to the determination of similarity transformations, not similarity solutions.

\section{The invariance condition}

Consider one-parameter Lie groups of the form

$$
\left.\begin{array}{rl}
x^{\prime} & =X(x, y, t, \psi ; \epsilon), \quad y^{\prime}=Y(x, y, t, \psi ; \epsilon), \\
t^{\prime} & =T(x, y, t, \psi ; \epsilon), \quad \psi^{\prime}=\Psi(x, y, t, \psi ; \epsilon) .
\end{array}\right\}
$$

The surface

$$
F(x, y, t, \psi)=0
$$


is said to be invariant under the transformation (3) if, upon application of (3), $F$ is mapped onto itself, so that

$$
F\left(x^{\prime}, y^{\prime}, t^{\prime}, \psi^{\prime}\right)=F(x, y, t, \psi) .
$$

This is equivalent to requiring that the function $F$ reads the same when unprimed variables are exchanged for primed ones. If we expand (3) about the identity element (which can always be arranged to be $\epsilon=0$ ) then the corresponding infinitesimal transformation results:

$$
\left.\begin{array}{rl}
x^{\prime} & =x+\epsilon[\partial X / \partial \epsilon]_{\epsilon=0}+O\left(\epsilon^{2}\right)=x+\epsilon \xi(x, y, t, \psi)+O\left(\epsilon^{2}\right), \\
y^{\prime}=y+\epsilon[\partial Y / \partial \epsilon]_{\epsilon=0}+O\left(\epsilon^{2}\right) & =y+\epsilon \rho(x, y, t, \psi)+O\left(\epsilon^{2}\right), \\
t^{\prime} & =t+\epsilon[\partial T / \partial \epsilon]_{\epsilon=0}+O\left(\epsilon^{2}\right)=t+\epsilon T(x, y, t, \psi)+O\left(\epsilon^{2}\right), \\
\psi^{\prime}=\psi+\epsilon[\partial \Psi / \partial \epsilon]_{\epsilon=0}+O\left(\epsilon^{2}\right) & =\psi+\epsilon \eta(x, y, t, \psi)+O\left(\epsilon^{2}\right) .
\end{array}\right\}
$$

Relations (6) are substituted into the left-hand side of (5) and the result is expanded again for small $\epsilon$. If we define the differential operator $U$ as

then the result is

$$
U \equiv \xi \frac{\partial}{\partial x}+\rho \frac{\partial}{\partial y}+\tau \frac{\partial}{\partial t}+\eta \frac{\partial}{\partial \psi}
$$

$$
F(x+\epsilon \xi, y+\epsilon \rho, t+\epsilon \tau, \psi+\epsilon \eta)=F(x, y, t, \psi)+\epsilon U F+\frac{\epsilon^{2}}{2 !} U^{2} F+\ldots+\frac{\epsilon^{j}}{j !} U^{j} F+\ldots
$$

Thus a necessary and sufficient condition for (5) to be satisfied is

$$
U F=0 .
$$

Equation (9) is the general first-order partial differential equation of a surface $F$ which is invariant under the group (3).

\section{Invariant groups of (1)}

In the present work, the invariant groups of (1) were determined using the method of infinitesimal transformations set forth in Bluman \& Cole (1974, part 2). Since the method is well established, only the results will be stated here with some further details included in the appendix.

For the case $v=0,(1)$ is found to be invariant under a ten-parameter group with infinitesimals

$$
\left.\begin{array}{rl}
\xi & =a x+b y+c t y+f_{1}(t)+d, \\
\rho & =-b x+a y-c t x+f_{2}(t)+e, \\
\tau & =a k t+h, \\
\eta & =a(2-k) \psi+\frac{1}{2} c\left(x^{2}+y^{2}\right)-\dot{f}_{2}(t) x+\dot{f}_{1}(t) y+s(t)+p
\end{array}\right\}
$$

where a dot denotes differentiation with respect to time. The infinitesimals for the full viscous equation, $\nu=$ constant, are identical to (10) with $k$ set equal to 2 . This group has seven explicit parameters, $a, b, c, d, e, h$ and $p$, and three implicit parameters contained in the arbitrary once-differentiable functions of time $f_{1}(t), f_{2}(t)$ and $s(t)$. The constant $k$, which becomes an exponent in the finite stretching transformation associated with the parameter $a$ (see $\S 6$, example 3 ), is actually an eighth explicit parameter for the case $v=0$, although it does not generate a finite transformation of 
its own. The parameters $d, e$ and $p$ are not essential and could be combined with the arbitrary functions of time. See the appendix for some of the details leading up to (10).

Finite groups may be generated from the infinitesimals by means of a power-series expansion. Equation (8) showed that the change in the value of a function $F$ under the transformation (3) could be expressed in terms of a power series in the operator $U$ acting on $F$. Consider the finite group corresponding to the parameter $c$. Let $F=x$, $U x=t y, U(U x)=-t^{2} x$ etc. The series (8) then becomes

$$
x^{\prime}=x+y\left(c t-\frac{(c t)^{3}}{3 !}+\ldots\right)+x\left(-\frac{(c t)^{2}}{2 !}+\frac{(c t)^{4}}{4 !}-\ldots\right)=x \cos (c t)+y \sin (c t) .
$$

Similarly,

$$
y^{\prime}=-x \sin (c t)+y \cos (c t), \quad t^{\prime}=t, \quad \psi^{\prime}=\psi+\frac{1}{2} c\left(x^{2}+y^{2}\right) .
$$

The parameter $c$ corresponds to a transformation to a set of co-ordinates rotating at a constant angular velocity. The rest of the parameters in (10) form finite groups as follows:

$$
\begin{aligned}
a, & \text { invariance under stretching ( } k \text {-exponent), } \\
b, & \text { invariance under rotation of co-ordinates, } \\
d, e, h, p, & \text { invariance under translations in } x, y, t \text { and } \psi, \\
s(t), & \text { addition of an arbitrary function of time to } \psi, \\
f_{1}(t), f_{2}(t), & \text { invariance under non-uniform translation. }
\end{aligned}
$$

The finite transformation corresponding to $f_{1}(t), f_{2}(t)$ and $s(t)$ is

$$
\left.\begin{array}{c}
x^{\prime}=x+f_{1}(t), \quad y^{\prime}=y+f_{2}(t), \quad t^{\prime}=t, \\
\psi^{\prime}=\psi-\dot{f}_{2} x+\dot{f}_{1} y+s(t) .
\end{array}\right\}
$$

Invariance of (1) under the group (11) may be easily checked by direct substitution. The function $s(t)$ can be chosen such that (11) is invariant under the binary operation of composition. That is, the result of applying two successive transformations, e.g. $\left(f_{1}, f_{2}\right)$ followed by, say, $\left(h_{1}, h_{2}\right)$, is equivalent to applying the single transformation $\left(f_{1}+h_{1}, f_{2}+h_{2}\right) \dagger$

The set of transformations (11) allows us to describe an unsteady flow in terms of a co-ordinate system which convects non-uniformly. This means that we can 'think Lagrangian' without actually having to resort to a Lagrangian formulation of the equations. In a flow composed of definable vortex structures, we can always think in terms of a co-ordinate system which remains attached to some preferred point in a structure such as a peak in the vorticity field or a minimum in the pressure field. Within such a co-ordinate system we may look for similarity in terms of any of the available groups of $(10)$.

$\dagger$ In moving co-ordinates, the functions $f_{1}$ and $f_{2}$ in (11) enter the momentum equation as virtual body forces $\ddot{f}_{1}$ and $\ddot{f}_{2}$. For this reason invariance of the momentum equation holds only for a Galilean transformation. However, the operation of taking the curl to get the vorticity equation causes these forces to drop out. Therefore both the vorticity equation and the streamfunction equation are invariant in an accelerating frame. 


\section{Construction of similarity variables}

Equation (1) is a surface in a 38-dimensional space which is left invariant under the transformations (plus extensions; see appendix) of a ten-parameter group whose infinitesimals are given by (10). It is now necessary to show how these infinitesimals can be combined with the results of $\S 3$ to construct four-dimensional surfaces $F(x, y, t, \psi)$ which are invariant under the same group.

The function $F$ must satisfy the invariance condition (9), which we write as

$$
\xi \frac{\partial F}{\partial x}+\rho \frac{\partial F}{\partial y}+\tau \frac{\partial F}{\partial t}+\eta \frac{\partial F}{\partial \psi}=0
$$

This equation can be replaced by its characteristic equations

$$
\frac{d x}{\xi}=\frac{d y}{\rho}=\frac{d t}{\tau}=\frac{d \psi}{\eta} .
$$

In principle, the general solution to (12) can be found. Since the infinitesimals $\xi, \rho$ and $\tau$ are independent of $\psi$, the solution will involve three constants of integration (three invariant surfaces). The first two are obtained by solving the first two equalities, and they become the similarity variables $\theta(x, y, t)$ and $\phi(x, y, t)$. The third constant is obtained by solving the last equality and plays the role of a dependent variable $F(\theta, \phi)$. The order of the equalities in (12) can be taken in several possible ways. Suppose that $\theta(x, y, t)$ is the integral curve of the equality involving $\xi$ and $\tau$ and that $\phi(x, y, t)$ is the integral curve of the equality involving $\rho$ and $\tau$. Then $x$ and $y$ can each be expressed in terms of $\theta, \phi$ and $t$, say $x=\sigma(\theta, \phi, t)$ and $y=\omega(\theta, \phi, t)$. Then the last equality becomes

$$
d t / \tau(t)=d \psi / \eta(\sigma, \omega, t, \psi)
$$

with the solution $G(\theta, \phi, t, \psi)=$ constant $=F(\theta, \phi)$, where $\theta$ and $\phi$ are treated as constant during the integration and the function $G$ is known explicitly. Solving for $\psi$ gives

$$
\psi=g(x, y, t, F(\theta, \phi))
$$

Equation (13) is equivalent to (2) with $\theta$ and $\phi$ replaced by their explicit representations in terms of $x, y$ and $t$. Substitution of (13) into (1) will accomplish the required reduction of variables.

\section{Transformations}

In this section we shall derive some of the similarity transformations which (1) will admit. It should be emphasized that the phrase 'similarity transformation' applies to any functional form for $\psi$ which accomplishes our purpose of reducing the number of independent variables in (1) from three to two. Hence invariance under translations in $x, y$ and $t$ (parameters $d, e$ and $h$ ) leads to the simple forms $\psi(y, t), \psi(x, t)$ and $\psi(x, y)$. Invariance under rotation (parameter $b$ ) leads to $\psi\left(x^{2}+y^{2}, t\right)$.

Generally speaking, the similarity transformations for (1) fall into two classes depending on whether or not the stretching parameter $a$ and time translation parameter $h$ are equal to zero. If both parameters are zero, then $\tau=0$, and $t$ becomes one of the similarity variables, the other being some function $\theta(x, y, t)$. The stream function 
takes the form $\psi=H(x, y, t)+F(\theta, t)$, and the reduced equation for $F$ is linear. When the parameter $a$ is not equal to zero then both similarity variables depend on the space co-ordinates and the reduced equation for $F$ is nonlinear.

Examples 1 and 2 will illustrate transformations of the first type and examples 3 and 4 will illustrate transformations of the second type.

Example 1. Invariance under a transformation to a co-ordinate system which moves in an arbitrary irrotational fashion.

(a) Infinitesimals:

$$
\xi=f_{1}(t), \quad \rho=f_{2}(t), \quad \tau=0, \quad \eta=-\dot{f}_{2} x+\dot{f}_{1} y .
$$

(b) Characteristic equations:

$$
\frac{d x}{f_{1}(t)}=\frac{d y}{f_{2}(t)}=\frac{d t}{0}=\frac{d \psi}{-\dot{f}_{2} x+\dot{f}_{1} y}
$$

(c) Similarity variables. Note that if either $f_{1}$ or $f_{2}$ is zero then the similarity variables become $(x, t)$ or $(y, t)$. Integration of the characteristic equations leads to

$$
\theta=x / f_{1}-y / f_{2}, \quad \phi=t .
$$

We can compute $\psi$ in two ways:

$$
\psi=\int_{x_{0}}^{x}\left(-\frac{\dot{f}_{2}}{f_{1}} x-\frac{f_{2} \dot{f}_{1}}{f_{1}} \theta+\frac{f_{2} \dot{f}_{1}}{f_{1}^{2}} x\right) d x+W(\theta, t)
$$

or

$$
\psi=\int_{y_{0}}^{y}\left(-\frac{f_{1} \dot{f}_{2}}{f_{2}^{2}} y-\frac{\dot{f}_{2} f_{1}}{f_{2}} \theta+\frac{\dot{f_{1}}}{f_{2}} y\right) d y+S(\theta, t)
$$

Retaining the obvious symmetry in $x$ and $y$ (with $\alpha, \beta$ constants) gives

$$
\begin{aligned}
\psi= & -\frac{\alpha}{2(\alpha+\beta)}\left(\frac{f_{1} \dot{f}_{2}-f_{2} \dot{f}_{1}}{f_{1}^{2}}\right)\left(x^{2}-x_{0}^{2}\right)-\frac{\beta}{2(\alpha+\beta)}\left(\frac{f_{1} \dot{f}_{2}-f_{2} \dot{f}_{1}}{f_{2}^{2}}\right)\left(y^{2}-y_{0}^{2}\right) \\
& -\frac{\alpha}{(\alpha+\beta)} \frac{\dot{f}_{1} f_{2}}{f_{1}^{2}}\left(x^{2}-x_{0} x\right)-\frac{\beta}{(\alpha+\beta)} \frac{\dot{f}_{2}}{f_{2}}\left(x y-y_{0} x\right) \\
& +\frac{\alpha}{(\alpha+\beta)} \frac{\dot{f}_{1}}{f_{1}}\left(x y-x_{0} y\right)+\frac{\beta}{(\alpha+\beta)} \frac{\dot{f}_{2} f_{1}}{f_{2}^{2}}\left(y^{2}-y_{0} y\right)+F(\theta, t) .
\end{aligned}
$$

(d) Reduced equation. Substitution of (14) into (1) gives

$$
H_{1}(t)+H_{2}(t) \theta G_{\theta}+G_{t}=\nu H_{3}(t) G_{\theta \theta},
$$

where

$$
\begin{gathered}
H_{1}=-\frac{1}{(\alpha+\beta)} \frac{d}{d t}\left\{\left(\frac{\alpha}{f_{1}^{2}}-\frac{\beta}{f_{2}^{2}}\right) \frac{d}{d t}\left(f_{1} f_{2}\right)\right\}, \\
H_{2}=-\frac{1}{f_{1} f_{2}} \frac{d}{d t}\left(f_{1} f_{2}\right), \quad H_{3}=\left(\frac{1}{f_{1}^{2}}+\frac{1}{f_{2}^{2}}\right) \\
G=\left(\frac{1}{f_{1}^{2}}+\frac{1}{f_{2}^{2}}\right) F_{\theta \theta} .
\end{gathered}
$$

and

At any given instant the curves along which the similarity variable $\theta$ is constant are straight lines in the $x, y$ plane whose slope and intercept are defined by the values of $f_{1}$ and $f_{2}$ (see figure 1 ). 


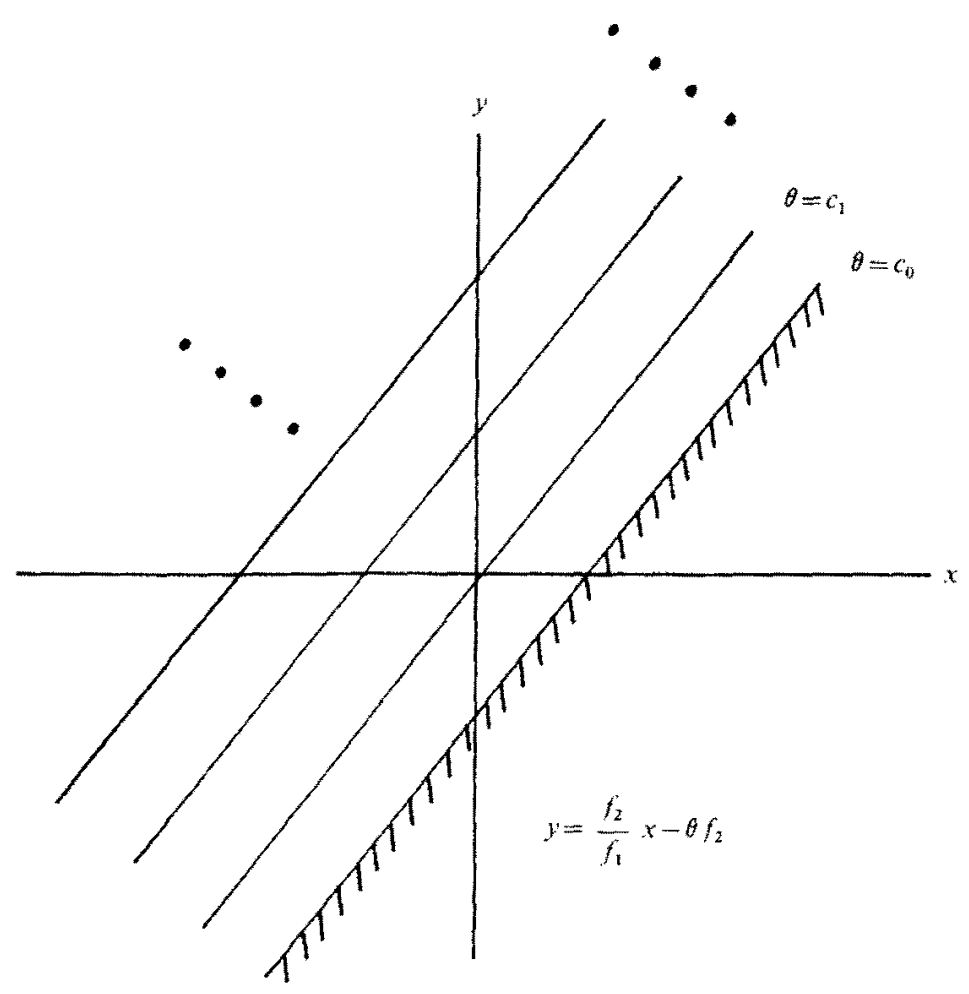

Figure 1

Similarity transformations can be produced from the combination of two or more groups.

Example 2. Combine a rotation with a non-uniform translation.

(a) Infinitesimals:

$$
\xi=y+f_{1}(t), \quad \rho=-x, \quad \tau=0, \quad \eta=f_{1}(t) y .
$$

(b) Characteristic equations:

$$
\frac{d x}{y+f_{1}(t)}=\frac{d y}{-x}=\frac{d t}{0}=\frac{d \psi}{\dot{f}_{1}(t) y}
$$

(c) Similarity variables. Integration of the above equations gives

$$
\theta=x^{2}+\left(y+f_{1}\right)^{2}-f_{1}^{2}, \quad \phi=t .
$$

Again we can define $\psi$ in two ways. Combining both gives

$$
\psi=f_{1} x+\frac{f_{1} f_{1}}{2}\left\{\sin ^{-1}\left(\frac{y+f_{1}}{\left(x^{2}+\left(y+f_{1}\right)^{2}\right)^{\frac{1}{2}}}\right)-\sin ^{-1}\left(\frac{x}{\left(x^{2}+\left(y+f_{1}\right)^{2}\right)^{\frac{1}{2}}}\right)\right\}+F(\theta, t) .
$$

At any given instant, the lines $\theta=$ constant form a set of concentric circles in the $x, y$ plane with radii $\left(f_{1}^{2}+\theta\right)^{\frac{1}{2}}$ and centres at $(x, y)=\left(0,-f_{1}\right)$ (see figure 2 ).

(d) Reduced equation. Substitution of (16) into (1) leads to

$$
G_{t}=4 \nu \partial\left[\left(\theta+f_{1}^{\mathrm{S}}\right) G_{\theta}\right] / \partial \theta
$$

where

$$
G=\partial\left[\left(\theta+f_{1}^{2}\right) F_{\theta}\right] / \partial \theta
$$




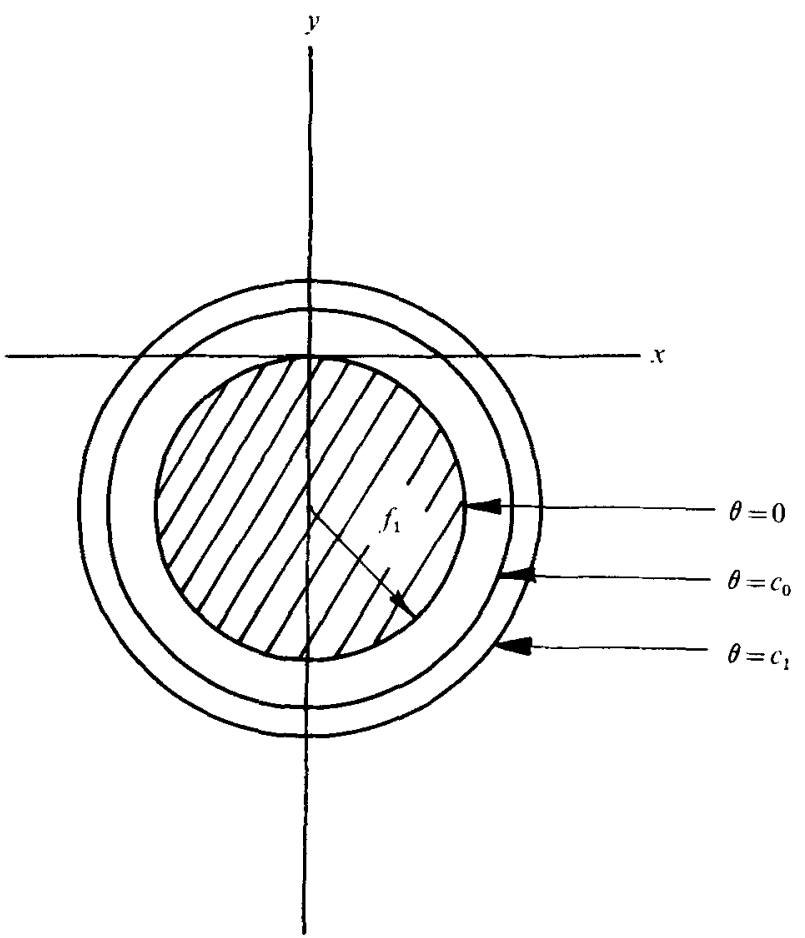

Figure 2

Example 3. Invariance under stretching.

(a) Infinitesimals:

(b) Finite group:

$$
\xi=x, \quad \rho=y, \quad \tau=k t, \quad \eta=(2-k) \psi .
$$$$
x^{\prime}=a x, \quad y^{\prime}=a y, \quad t^{\prime}=a^{k} t, \quad \psi^{\prime}=a^{(2-k)} \psi .
$$

(c) Characteristic equations:

(d) Similarity variables:

$$
\frac{d x}{x}=\frac{d y}{y}=\frac{d t}{k t}=\frac{d \psi}{(2-k) \psi} .
$$

$$
\theta=x / t^{1 / k}, \quad \phi=y / t^{1 / k}, \quad \psi=t^{(2-k) / k} F(\theta, \phi) .
$$

(e) Reduced equation. Substitution of (18) into (1) gives

where

$$
-\nabla^{2} F+\left(F_{\phi}-\theta / k\right) \nabla^{2} F_{\theta}-\left(F_{\theta}+\phi / k\right) \nabla^{2} F_{\phi}=0,
$$

The reduced viscous equation $(k=2)$ is

$$
-\nabla^{2} F+\left(F_{\phi}-\frac{1}{2} \theta\right) \nabla^{2} F_{\theta}-\left(F_{\theta}+\frac{1}{2} \phi\right) \nabla^{2} F_{\phi}=\nu \nabla^{4} F .
$$

Example 4. The general similarity transformation for (1).

(a) Infinitesimals. Divide (10) through by $a$ and relabel the parameters:

$$
\begin{gathered}
\xi=x+b y+c t y+f_{1}(t), \quad \rho=-b x+y-c t x+f_{2}(t), \\
\tau=k t, \quad \eta=(2-k) \psi+\frac{1}{2} c\left(x^{2}+y^{2}\right)-\dot{f}_{2}(t) x+\dot{f}_{1}(t) y .
\end{gathered}
$$


(b) Characteristic equations:

$$
\frac{d x}{x+b y+c t y+f_{1}(t)}=\frac{d y}{-b x+y-c t x+f_{2}(t)}=\frac{d t}{k t}=\frac{d \psi}{(2-k) \psi+\frac{1}{2} c\left(x^{2}+y^{2}\right)-f_{2}^{\prime}(t) x+\dot{f_{1}(t) y}}
$$

(c) Similarity variables. We need to solve the first two characteristic equations for $x$ and $y$. The two constants of integration that are generated will become the similarity variables. The problem involves solving the homogeneous adjoint system corresponding to the first two equalities via a series expansion, from which the solution to the inhomogeneous system follows directly. The result after a considerable amount of manipulation is

$$
\begin{aligned}
& \theta=t^{-1 / k}\left[(x-X(t)) \cos \left(\frac{b \ln t+c t}{k}\right)-(y-Y(t)) \sin \left(\frac{b \ln t+c t}{k}\right)\right], \\
& \phi=t^{-1 / k}\left[(x-X(t)) \sin \left(\frac{b \ln t+c t}{k}\right)+(y-Y(t)) \cos \left(\frac{b \ln t+c t}{k}\right)\right],
\end{aligned}
$$

and the expression for $\psi$ is

$$
\psi=t^{(2-k) / k} F(\theta, \phi)+\frac{c}{2 k}\left(x^{2}+y^{2}\right)-x\left[\dot{Y}(t)+\frac{c}{k} X(t)\right]+y\left[\dot{X}(t)-\frac{c}{k} Y(t)\right] .
$$

At intermediate stages in the calculation, $X(t)$ and $Y(t)$ are expressed in terms of $f_{1}(t)$ and $f_{2}(t)$; however, these expressions become superfluous when the final form (20) is reached.

(d) Reduced equation. Substitution of (20) into (1) gives

where

$$
-\nabla^{2} F+\left[F_{\phi}-(\theta+b \phi) / k\right] \nabla^{2} F_{\theta}-\left[F_{\theta}+(\phi-b \theta) / k\right] \nabla^{2} F_{\phi}=0,
$$

The reduced viscous equation $(k=2)$ is

$$
-\nabla^{2} F+\left[F_{\phi}-\frac{1}{2}(\theta+b \phi)\right] \nabla^{2} F_{\theta}-\left[F_{\theta}+\frac{1}{2}(\phi-b \theta)\right] \nabla^{2} F_{\phi}=\nu \nabla^{4} F .
$$

The system (20) describes a fairly general unsteady flow in which there may be:

(i) diffusion according to $t^{1 / k}$,

(ii) non-uniform translation according to $X(t)$ and $Y(t)$,

(iii) uniform rotation at angular velocity $c$,

(iv) rotation at an ever decreasing rate according to $b \ln t$.

The non-uniform translation and uniform rotation could be introduced directly into the transformation (20) from a knowledge of the groups of (10). However, the $\ln t$ term is new and can be found only by integrating the characteristic equations.

\section{Particle paths}

Consider unsteady motion of a fluid in two dimensions. The pathlines of fluid particles are described by the parametric equations

$$
\begin{aligned}
& x(t)=x_{0}+\int_{t_{0}}^{t} u(x(t), y(t), t) d t, \\
& y(t)=y_{0}+\int_{t_{0}}^{t} v(x(t), y(t), t) d t,
\end{aligned}
$$


where $u$ and $v$ are the velocity components running with the particle, and $t$ is the parameter along a pathline. The constants $\left(x_{0}, y_{0}, t_{0}\right)$ serve to label the particle. In terms of differentials,

$$
d x / d t=u(x, y, t), \quad d y / d t=v(x, y, t) .
$$

In general, the solution of the above equations may present considerable analytical difficulties. This is particularly so if the functions $u$ and $v$ are defined empirically on a grid of data points. However, if the flow can be approximated by a similarity form for the stream function then the particle-path equations can be reduced to an autonomous system.

Consider the general form of example 4. Rewrite (22) in terms of the similarity variables $\theta, \phi$ and $F$. The result after some straightforward manipulations is

where $\tau=\ln t$.

$$
\left.\begin{array}{c}
d \theta / d \tau=F_{\phi}-(\theta+b \phi) / k, \\
d \phi / d \tau=-\left[F_{\theta}+(\phi-b \theta) / k\right],
\end{array}\right\}
$$

The value of such a reduction is clear. The solution curves of system (23) form a family in the $\theta, \phi$ plane. Thus, while the co-ordinates of a given particle may vary with time, the slopes of particle trajectories in the $\theta, \phi$ plane are independent of time. Particle trajectories never cross except at the critical points (stagnation points, points of fluid accumulation) of system (23). Thus the entrainment process for a flow which can be described by (20) can be characterized completely in terms of the critical points and isoclines of system (23).

To see how this might work, consider the case $b=c=0$. The similarity form (20) reduces to stretching and non-uniform translation. The functions $X(t)$ and $Y(t)$ are restricted only by the requirement that they be once differentiable; otherwise they are arbitrary, and may be used to describe the convective motion of an eddy which diffuses according to $t^{1 / k}$ while maintaining its geometric shape $F(\theta, \phi)$ and orientation. In the most general case, the streamline pattern of the eddy may undergo a rotation with phase angle $(b \ln t+c t) / k$ while convecting and diffusing. The constants $b, c$ and $k$ and functions $X(t)$ and $Y(t)$ are all available to fit the functional form (20) to the particular flow in question. In a flow composed of an array of eddies, these ideas may apply locally if the eddies do not interact too strongly. In a mixing layer, for example, a large-scale eddy may persist for a certain time before disappearing in a process of amalgamation with other eddies to form an eddy of even larger scale (Brown \& Roshko 1974). One may hypothesize that for the early part of its lifetime, the flow in the neighbourhood of the eddy may be approximately described by a stream function of the form of (20). Over the same period, entrainment in the neighbourhood of the eddy would be described by a phase-plane plot of particle paths. Such an entrainment diagram would be especially useful for understanding how and where fluid is mixed by the large-scale motions in a turbulent shear flow.

There is at least one precedent in the literature of turbulence where this approach has been used to describe entrainment. This is Turner's paper (1964, figure 4) on the turbulent thermal, in which a similarity form is applied to a rising and expanding Hill's spherical vortex. In a recent experiment, Cantwell et al. (1977) were able to fit a similarity form to measurements on the plane of symmetry of a turbulent spot. The resulting phase-plane plot of particle trajectories was used to obtain quantitative information about entrainment along the turbulent interface of the spot. 


\section{Concluding remarks}

The infinitesimals of the general invariance group of the two-dimensional, unsteady, stream-function equation have been derived and a few similarity transformations have been described by which the number of independent variables in the equation may be reduced by one. The new equation which results from such a transformation is a partial differential equation in two independent variables which, like any partial differential equation, may admit an infinite number of solutions, thus precluding a general discussion of similarity solutions. Rather, the effort here was directed towards a more fundamental understanding of the unsteady motion of an incompressible fluid in two dimensions, particularly with regard to the problem of solving for particle paths. A hypothesis is put forth regarding a diagrammatical approach to the problem of entrainment in fluid flows dominated by large-scale, two-dimensional, coherent vortex motions. Stated simply, the hypothesis is this: given measurements or computations of a two-dimensional unsteady flow field, try to fit a stream function of the form (20) with $b, c, k, X(t)$ and $Y(t)$ chosen for a best fit. If good agreement can be found over a certain region, then within that region particle trajectories may be described in terms of the isoclines and critical points of an autonomous set of ordinary differential equations.

I should like to express thanks to Prof. A. E. Perry, who first convinced me of the value of phase-plane diagrams for interpreting fluid flows. This work was supported, in part, by the National Science Foundation.

\section{Appendix. Determination of infinitesimals}

The invariance condition (9) can be extended to higher dimensions, and the higherdimensional surface we have in mind is (1). Our objective here is reversed from what it was in $\S 5$. There the infinitesimals $\xi, \rho, \tau$ and $\eta$ were known, and the invariant surface was unknown. Here the surface (1) is known, and the infinitesimals are assumed to be unknown. In this context, (1) is thought of as an algebraic relation between the various partial derivatives of $\psi$. It is a surface in a 38-dimensional space. The search for invariant groups of (1) proceeds with 38-dimensional groups expanded in the form of (6) and an invariance condition (9) with 38 terms (only 11 are non-zero).

Since all but four of the co-ordinates in this space are partial derivatives in the four basic variables $x, y, t$ and $\psi$, the infinitesimal transformations for those co-ordinates turn out to be written in terms of the unknown infinitesimals $\xi, \rho, \tau$ and $\eta$. This important fact makes it possible to use the invariance condition to generate a large number of linear partial differential equations which are sufficient to determine $\xi, \rho, \tau$ and $\eta$.

First it is necessary to extend the group (3) to include transformations of all of the required derivatives of $\psi$. Assume that (3) has been so chosen that the invariance condition (9) is satisfied for the surface $F(x, y, t, \psi)=0$, where $F$ is of the form

$$
F(x, y, t, \psi)=g(x, y, t)-\psi
$$

and $g(x, y, t)$ is a solution of (1). Replace $\psi$ in (3) by $g$ and make the correspondence $(x, y, t) \leftrightarrow\left(x_{1}, x_{2}, x_{3}\right),(\xi, \rho, \tau) \leftrightarrow\left(\xi_{1}, \xi_{2}, \xi_{3}\right)$. The infinitesimal transformations (6) become

$$
\begin{gathered}
x_{i}^{\prime}=x_{i}+\epsilon \xi_{i}\left(x_{1}, x_{2}, x_{3}, g\right)+O\left(\epsilon^{2}\right) \quad(i=1,2,3), \\
\psi^{\prime}=g\left(x_{1}, x_{2}, x_{3}\right)+\epsilon \eta\left(x_{1}, x_{2}, x_{3}, g\right)+O\left(\epsilon^{2}\right) .
\end{gathered}
$$


From $(24 b)$ we have

$$
\left.\frac{\partial \psi^{\prime}}{\partial x_{j}^{\prime}}=\left[\frac{\partial g}{\partial x_{i}}+\epsilon\left(\frac{\partial \eta}{\partial x_{i}}+\frac{\partial \eta}{\partial \psi} \frac{\partial g}{\partial x_{i}}\right)\right] \frac{\partial x_{i}}{\partial x_{j}^{\prime}} \quad \text { (sum over } i\right)
$$

The nine auxiliary derivatives $\partial x_{i} / \partial x_{j}^{\prime}$ are taken with $\psi=g\left(x_{1}, x_{2}, x_{3}\right)$ and the remaining primed independent variables $x_{m}^{\prime}(m \neq j)$ held fixed. From $(24 a)$,

$$
\frac{\partial x_{i}}{\partial x_{j}^{\prime}}=\delta_{i j}-\epsilon\left(\frac{\partial \xi_{i}}{\partial x_{k}}+\frac{\partial \xi_{i}}{\partial \psi} \frac{\partial g}{\partial x_{k}}\right) \frac{\partial x_{k}}{\partial x_{j}^{\prime}}+O\left(\epsilon^{2}\right) \quad \text { (sum over } k \text { ), }
$$

where $\delta_{i j}$ is the Kronecker delta. From (24) we see that two of the terms in the summation in (26) will be of order $\epsilon^{2}$. With these two terms dropped, the remaining terms in (26) are rearranged and expanded again for small $\epsilon$ (for cross-derivatives, this rearrangement is unnecessary) to give

$$
\frac{\partial x_{i}}{\partial x_{j}^{\prime}}=\delta_{i j}-\epsilon\left(\frac{\partial \xi_{i}}{\partial x_{j}}+\frac{\partial \xi_{i}}{\partial \psi^{\prime}} \frac{\partial g}{\partial x_{j}}\right)
$$

When the auxiliary derivatives (27) are substituted into (25) and terms of order $\epsilon$ are retained, the result is

where $N_{x_{j}}$ is given by

$$
\begin{gathered}
\partial \psi^{\prime} / \partial x_{j}^{\prime}=\partial g / \partial x_{j}+\epsilon N_{x_{j}}+O\left(\epsilon^{2}\right) \\
\left.N_{x_{j}}=\frac{D \eta}{D x_{j}}-\frac{D \xi_{k}}{D x_{j}} \frac{\partial g}{\partial x_{k}} \quad \text { (sum over } k\right)
\end{gathered}
$$

and the total differential operator is defined as

$$
\frac{D}{D x_{j}}=\frac{\partial}{\partial x_{j}}+\frac{\partial g}{\partial x_{j}} \frac{\partial}{\partial \psi} .
$$

The function $N_{x_{j}}$ is called the first extension of the group with respect to $x_{j}$. Second extensions refer to the way second partial derivatives transform, third extensions refer to third partial derivatives, etc. It is well to remember that the subscripts on the $N$ 's are labels and do not denote differentiation. Relation (28) is one we can add to the list of infinitesimal transformations (24). Bluman \& Cole (1974) give a detailed generalized account of the apparatus needed for computing the infinitesimals of the extended group. In our case, we need consider only one dependent variable. To compute the $n$th extension, one applies the total differential operator $D / D_{x_{j_{n}}}$ to the $(n-1)$ th extension $N_{j_{1} j_{2} j_{3} \cdots j_{n-1}}$ (each $j$ may refer to any one of the independent variables) and adds the term

$$
-\frac{D \xi_{k}}{D x_{j_{n}}} \frac{\partial^{n} g}{\partial x_{j_{1}} \partial x_{j_{2}} \ldots \partial x_{j_{n-1}} \partial x_{k}} \quad \text { (sum over } k \text { ). }
$$

We can see from (1) that the extensions $N_{x}, N_{y}, N_{x x t}, N_{y y t}, N_{x x x}, N_{x x y}, N_{y y y}, N_{y y x}$, $N_{x x x x}, N_{x x y y}$ and $N_{y y y y}$ will be required. Beyond the second order, the expressions become extremely long and would add little to the content of this paper except length. Notice that the extensions are always linear in the various derivatives of the four unknown functions $\xi, \rho, \tau$ and $\eta$.

Equation (1) is the surface

$$
S\left(g_{x}, g_{y}, g_{x x t}, g_{y y t}, g_{x x x}, g_{x y y}, g_{x x y}, g_{y y y}, g_{x x x x}, g_{x, c y y}, g_{y y y y}\right)=0
$$


and for invariance it must satisfy (9), where the operator includes the infinitesimals for the multiply extended group. In other words, the expanded transformation group includes (24) plus expressions for the first extensions (28),

$$
\psi_{x^{\prime}}^{\prime}=g_{x}+\epsilon N_{x}, \psi_{y^{\prime}}^{\prime}=g_{y}+\epsilon N_{y}
$$

third extensions $\psi_{x^{\prime} x^{\prime} t^{\prime}}^{\prime}=g_{x x t}+\epsilon N_{x x t}$ and so on. The invariance condition becomes

$$
\begin{aligned}
& N_{x} \frac{\partial S}{\partial g_{x}}+N_{y} \frac{\partial S}{\partial g_{y}}+N_{x x t} \frac{\partial S}{\partial g_{x x t}}+N_{y y t} \frac{\partial S}{\partial g_{y y t}}+N_{x x x} \frac{\partial S}{\partial g_{x x x}}+N_{y y x} \frac{\partial S}{\partial g_{y y x}} \\
& \quad+N_{x x y} \frac{\partial S}{\partial g_{x x y}}+N_{y y y} \frac{\partial S}{\partial g_{y y y}}+N_{x x x x} \frac{\partial S}{\partial g_{x x x x}}+N_{x x y y} \frac{\partial S}{\partial g_{x x y y}}+N_{y y y y} \frac{\partial S}{\partial g_{y y y y}}=0 .
\end{aligned}
$$

Equation (30) is of the form of (9). It is special only in the sense that the coefficients can be written in terms of the four basic functions $\xi, \rho, \tau$ and $\eta$. Note that if $S$ depended on $x, y, t, g$ or any other derivatives of $g$, say $g_{x x}$, then (30) would contain the corresponding terms $\xi(\partial S / \partial x), \rho(\partial S / \partial y), N_{x x}\left(\partial S / \partial g_{x x}\right)$, etc.

Differentiate (1) and substitute into (30) to get

$$
\begin{aligned}
\left(N_{t x x}+N_{t y y}\right)+g_{y}\left(N_{x x x}+N_{x y y}\right) & -g_{x}\left(N_{y x x}+N_{y y y}\right)+N_{y}\left(g_{x x x}+g_{x y y}\right) \\
& -N_{x}\left(g_{y x x}+g_{y y y}\right)-v\left(N_{x x x x}+2 N_{x x y y}+N_{y y y y}\right)=0 .
\end{aligned}
$$

Equation (31) plus expressions for the various extensions are the tools required for determining the four unknown functions $\xi, \rho, \tau$ and $\eta$. The strategy for this is as follows.

(1) Every term in (31) is of the form $A B$, where $A$ is some partial derivative of $\xi$, $\rho, \tau$ or $\eta$ and $B$ is a product of partial derivatives of $g$. Begin by gathering together terms which multiply the same combinations of derivatives of $g$.

(2) Attend to those combinations which are identical to those which appear in (1). All the combinations in (1) (with $\psi$ replaced by $g$ ) will appear in (31) and, for the coefficients which multiply these particular combinations, there are two possibilities which will permit (31) to be satisfied: either they are all zero [as must be true for the rest of the coefficients in (31)] or they are all equal (we choose the latter possibility), in which case they may be taken out as a common term multiplying (1). Equation (1) is, of course, equal to zero by the assumption that $g(x, y, t)$ is a solution.

(3) As was just stated in step 2, all the other coefficients multiplying the various combinations of derivatives of $g$ are set equal to zero.

(4) The result of steps 2 and 3 is a greatly overdetermined set of linear partial differential equations in the unknowns $\xi(x, y, t, \psi), \rho(x, y, t, \psi), \tau(x, y, t, \psi)$ and $\eta(x, y, t, \psi)$. Initial considerations of this large number of partial differential equations permit most of them to be eliminated, and only a very few play a role in ultimately determining $\xi, \rho, \tau$ and $\eta$. These are called the determining equations of the group. The following determining equations hold for both $\nu=0$ and $\nu \neq 0$ :

$$
\left.\begin{array}{c}
\tau_{x}=\tau_{y}=\xi_{\psi}=\rho_{\psi}=\tau_{\psi}=\eta_{y, \psi}=\eta_{x \psi}=\eta_{y \psi}=\xi_{x x}=\xi_{y y}=\xi_{x y}=\rho_{x x}=\rho_{y y}=\rho_{x y}=0 \\
\rho_{x}=-\xi_{y}, \quad \rho_{y}=\xi_{x}, \quad \eta_{x}=-\rho_{t}, \quad \eta_{y}=\xi_{t}, \quad \eta_{t \psi}=2 \xi_{t x}, \quad \eta_{t \psi}=2 \rho_{t y}, \\
\eta_{x x y}+\eta_{y y y}=\eta_{x x x}+\eta_{y y x}=0, \quad \eta_{\psi}+\tau_{t}-2 \xi_{x}=0 .
\end{array}\right\}
$$


When $\nu \neq 0$, we have in addition the relation

$$
\eta_{\psi}=0 \text {. }
$$

When the determining equations (32) and (33) are solved, the infinitesimals (10) result.

\section{REFERENCES}

Ames, W. F. 1972 Nonlinear Partial Differential Equations in Engineering, vol. 2, chap. 2. Academic Press.

Birkногт, G. 1950 Hydrodynamics. Princeton University Press.

Bluman, G. W. \& Cole, J. D. 1974 Similarity Methods for Differential Equations. Springer.

BRowaNd, F. K. 1975 Ensemble averaged large scale structure in the turbulent mixing layer. In Turbulent Mixing in Non-Reactive and Reactive Flows (ed. S. N. B. Murthy), pp. 317-322. Plenum.

Brown, G. L. \& Roshro, A. 1974 On density effects and large structure in turbulent mixing layers. J. Fluid Mech. 64, 775-816.

CANTWELI, B. J. 1975 A flying hot wire study of the turbulent near wake of a circular cylinder at a Reynolds number of 140000 . Ph.D. thesis, California Institute of Technology.

Cantwell, B.J., Coles, D. E. \& Dimotakis, P. E. 1977 Structure and entrainment on the plane of symmetry of a turbulent spot. Calif. Inst. Tech. Interim Rep. NSF Grant ENG7680150 .

CoHen, A. 1911 An Introduction to the Lie Theory of One Parameter Groups with Application to the Solution of Differential Equations. New York: G. E. Stechert (1931 Reprint).

COLES, D. E. \& Barker, S. J. 1975 Some remarks on a synthetic turbulent boundary layer. In Turbulent Mixing in Non-Reactive and Reactive Flows (ed. S. N. B. Murthy), pp. 285-294. Plenum.

FIEDLER, H. E. 1975 On turbulence structure and mixing mechanism in free turbulent shear flows. In Turbulent Mixing in Non-Reactive and Reactive Flows (ed. S. N. B. Murthy), pp. 381410. Plenum.

Hama, F. R. 1962 Streaklines in perturbed shear flow. Phys. Fluids 5, 644-650.

Hansex, A. G. 1964 Similarity Analysis of Boundary Value Problems in Engineering. PrenticeHall.

Ovsuannikov, L. V. 1962 Gruppovye Svoystva Differentsialny Uravneni. Novosibirsk (Group Properties of Differential Equations, trans. G. Bluman, 1967).

Rosen, G. \& ULLRICH, G. W. 1973 Invariance group of the equation $\partial \bar{u} / \partial t=-\bar{u} . \nabla \bar{u}$. SIAM J. Appl. Math. 24, 286-288.

STRUMPF, A. 1964 On a class of transformations leading to similar solutions of the steady two-dimensional Navier-Stokes equations. Ph.D. thesis, Stevens Institute of Technology.

Turner, J. S. 1964 The flow into an expanding spherical vortex. J. Fluid Mech. 18, 195-208.

WOODARD, H. S. \& AMES, W. F. 1971 Similarity solutions for partial differential equations generated by finite and infinitesimal groups. I.I.H.R. Rep. no. 132.

Wyananski, I., Sokolov, M. \& Friedman, D. 1976 On the turbulent spot in a boundary layer undergoing transition. J. Fluid Mech. 78, 785-819.

ZDRAVkovich, M. M. 1969 Smoke observations of the formation of a Kármán vortex street. J. Fluid Mech. 37, 491-496. 